\title{
Effect of Pore Connectivity on the Behavior of Fluids Confined in Sub-Nanometer Pores: Ethane and $\mathrm{CO}_{2}$ Confined in ZSM-22
}

\author{
Mohammed Musthafa Kummali ${ }^{1}$ (D), David Cole ${ }^{2}$ and Siddharth Gautam ${ }^{2, *(D)}$ \\ 1 Department of Physics, The New College (Autonomous), 147 Peters Road, Chennai 600014, India; \\ musthafakummali@thenewcollege.edu.in \\ 2 School of Earth Sciences, The Ohio State University, 125 South Oval Mall, Columbus, OH 43210, USA; \\ cole.618@osu.edu \\ * Correspondence: gautam.25@osu.edu
}

Citation: Kummali, M.M.; Cole, D.; Gautam, S. Effect of Pore

Connectivity on the Behavior of Fluids Confined in Sub-Nanometer Pores: Ethane and $\mathrm{CO}_{2}$ Confined in ZSM-22. Membranes 2021, 11, 113 https://doi.org/10.3390/membranes 11020113

Academic Editor: Tomohiro Kyotani Received: 27 December 2020

Accepted: 2 February 2021

Published: 5 February 2021

Publisher's Note: MDPI stays neutral with regard to jurisdictional claims in published maps and institutional affiliations.

Copyright: () 2021 by the authors. Licensee MDPI, Basel, Switzerland. This article is an open access article distributed under the terms and conditions of the Creative Commons Attribution (CC BY) license (https:// creativecommons.org/licenses/by/ $4.0 /)$.

\begin{abstract}
The behavior of fluids under nano-confinement varies from that in bulk due to an interplay of several factors including pore connectivity. In this work, we use molecular dynamics simulations to study the behavior of two fluids - ethane and $\mathrm{CO}_{2}$ confined in ZSM-22, a zeolite with channellike pores of diameter $0.55 \mathrm{~nm}$ isolated from each other. By comparing the behavior of the two fluids in ZSM-22 with that reported earlier in ZSM-5, a zeolite with pores of similar shape and size connected to each other via sinusoidal pores running perpendicular to them, we reveal the important role of pore connectivity. Further, by artificially imposing pore connectivity in ZSM-22 via inserting a 2-dimensional slab-like inter-crystalline space of thickness $0.5 \mathrm{~nm}$, we also studied the effect of the dimensionality and geometry of pore connectivity. While the translational motion of both ethane and $\mathrm{CO}_{2}$ in ZSM-22 is suppressed as a result of connecting the pores by perpendicular quasi-one-dimensional pores of similar dimensions, the effect of connecting the pores by inserting the inter-crystalline space is different on the translational motion of the two fluids. For ethane, pores connected via inter-crystalline space facilitate translational motion but suppress rotational motion, whereas in the case of $\mathrm{CO}_{2}$, both types of motion are suppressed by pore connection due to the strong interaction of $\mathrm{CO}_{2}$ with the surface of the substrate.
\end{abstract}

Keywords: $\mathrm{CO}_{2}$; ethane; zeolites; $\mathrm{MD}$ simulations; pore connectivity

\section{Introduction}

Zeolites are microporous materials which are commercially used as adsorbents in size and shape selective separation of molecular species and as catalysts in the petrochemical industry [1]. Due to their well-ordered pore structure, guest molecules adsorbed in zeolites exhibit behavior that differs considerably from the behavior of these molecules in their bulk systems [2]. This deviation of the behavior of guest molecules from the bulk is significantly enhanced for pores with smaller dimensions. This happens, for example, in zeolites like ZSM-5, which has a network of interconnected channel-like pores of diameter $0.55 \mathrm{~nm}$ $\left(0.56 \times 0.53 \mathrm{~nm}^{2}\right.$ in the $a-b$ plane running along $c$ axis) [3]. The strict geometrical restriction imposed by smaller pores of this dimension results in strong confinement of the guest molecules and leads to anomalous diffusion. This anomalous diffusion, which is mostly sub-diffusive, can in some severe cases even lead to single-file diffusion [4]. In addition to the geometrical restriction imposed by the nano-porous substrate, interactions between the guest molecule and the substrate can also put severe limitations on the diffusion of guest molecules. In particular, strong electrostatic interactions between the guest and the substrate can immobilize the guest molecules [5,6]. The presence of electrostatic interactions is responsible for the difference in the behavior of small hydrocarbons that are apolar and $\mathrm{CO}_{2}$ which has a quadrupole moment. This difference makes $\mathrm{CO}_{2}$ preferentially adsorb on a silica surface by displacing apolar hydrocarbons [7-10]. This strong preferential 
adsorption of $\mathrm{CO}_{2}$ due to its quadrupole moment also facilitates the separation of $\mathrm{CO}_{2}$ from a mixture of hydrocarbons [11,12].

The dynamics of guest molecules through nano-pores of a zeolite membrane is an important aspect of membrane technology. While the geometry and dimensions of the confining pores have a strong effect on the behavior of the guest molecules, the character of the inter-connectivity can also play an important role [13]. Although the effects of pore size and shape on the behavior of guest molecules have been extensively studied, the effects of pore connectivity remain relatively less explored. For this purpose, zeolites ZSM- 5 and ZSM-22 provide a very good opportunity because both have a network of similar channellike pores of $\sim 0.55 \mathrm{~nm}$ diameter oriented parallel to the crystallographic axis $b$ in ZSM- 5 and $c$ in ZSM-22, but with interconnecting pores in the $a-c$ plane in a sinusoidal arrangement in the former versus isolated channels in ZSM-22. A comparison of the behavior of guest molecules in ZSM- 5 and ZSM-22 can therefore reveal important insights on the role of pore connectivity in the behavior of guest molecules confined in sub-nanometer pores. Although the effects of pore connectivity involve a variety of other factors including the degrees of connectivity, pore volume, and surface areas, the simple comparison mentioned above can be a first step toward understanding the complex interplay between these factors.

To understand the role of electrostatic interactions and pore connectivity on the structure and dynamics of guest molecules confined in sub-nanometer pores of zeolitic materials, we report here MD simulation studies on ethane or $\mathrm{CO}_{2}$ confined in ZSM-22 pores. In this work, we study the different ways that the inter-connectivity of pores in a zeolite membrane may affect the structure and dynamics of typical guest molecules, thereby revealing important insights on this aspect of membrane technology. Comparative studies of the behavior of ethane and $\mathrm{CO}_{2}$ to understand the role of the quadrupole moment of $\mathrm{CO}_{2}$ have also been reported earlier [14,15]. The results obtained here for ZSM-22 are compared with those reported for ZSM-5 earlier [14] to understand the role of pore connectivity. Further, in some simulations reported here, the pores of ZSM-22 are connected artificially by inserting empty space connecting the pores. These simulations show that the quadrupole moment of $\mathrm{CO}_{2}$ and the pore connectivity of the substrate play an important role in determining the behavior of the confined species. We describe all the simulations carried out and reported here in Section 2, after which the salient results from them are listed in Section 3. In Section 4, we discuss the implications of the results and how they reveal the role of pore connectivity. Finally, we list the salient conclusions that can be made from the simulations in Section 5.

\section{Materials and Simulation Details}

The crystallographic unit cell of ZSM-22 has $24 \mathrm{Si}$ and $48 \mathrm{O}$ atoms. ZSM-22 has the Theta1 structure type and contains one-dimensional ten membered-ring pores (running along the Cartesian Z-direction) with channel diameters $4.5 \times 5.5 \AA^{2}$. ZSM-22 has an orthorhombic crystal structure with lattice constant $a=13.86, b=17.41$, and $c=5.04 \AA[16,17]$. In this work, a unit cell of ZSM-22 was replicated $3 \times 2 \times 6$ times using the visualization software VESTA [18] to get the starting simulation cell. Ethane or $\mathrm{CO}_{2}$ molecules were loaded into the pores of ZSM-22 in this cell using grand canonical Monte Carlo (GCMC) simulations. $\mathrm{MD}$ simulations were performed for three different loadings of ethane and $\mathrm{CO}_{2}$ molecules viz. 24, 52, and 72 molecules with a minimum of two, four, and six molecules in each pore. Periodic boundary conditions were applied in all directions during the simulations. Further, an additional simulation was performed at 52 molecular loading of $\mathrm{CO}_{2}$ by excluding the Coulombic interactions. Thus, a total of seven simulations were done, three for ethane and four for $\mathrm{CO}_{2}$. In addition, to investigate the effects of pore connectivity, an empty space of $2.5 \AA$ was inserted on the top and bottom of the ZSM-22 cell in the $Z$-direction, thereby exposing the pores to each other. With periodic boundary conditions applied, this renders the pores of ZSM-22 connected to each other via a 2-dimensional intercrystalline space of thickness $5 \AA$. Simulations with a loading of 72 ethane or $\mathrm{CO}_{2}$ molecules were then carried out in this modified ZSM-22 simulation cell with inter-crystalline space 
inserted. The highest loading of 72 molecules was considered for this simulation to increase the probability of the guest molecules migrating across the channels. Simulation snapshots showing the unmodified and modified cells are shown in Figure 1.
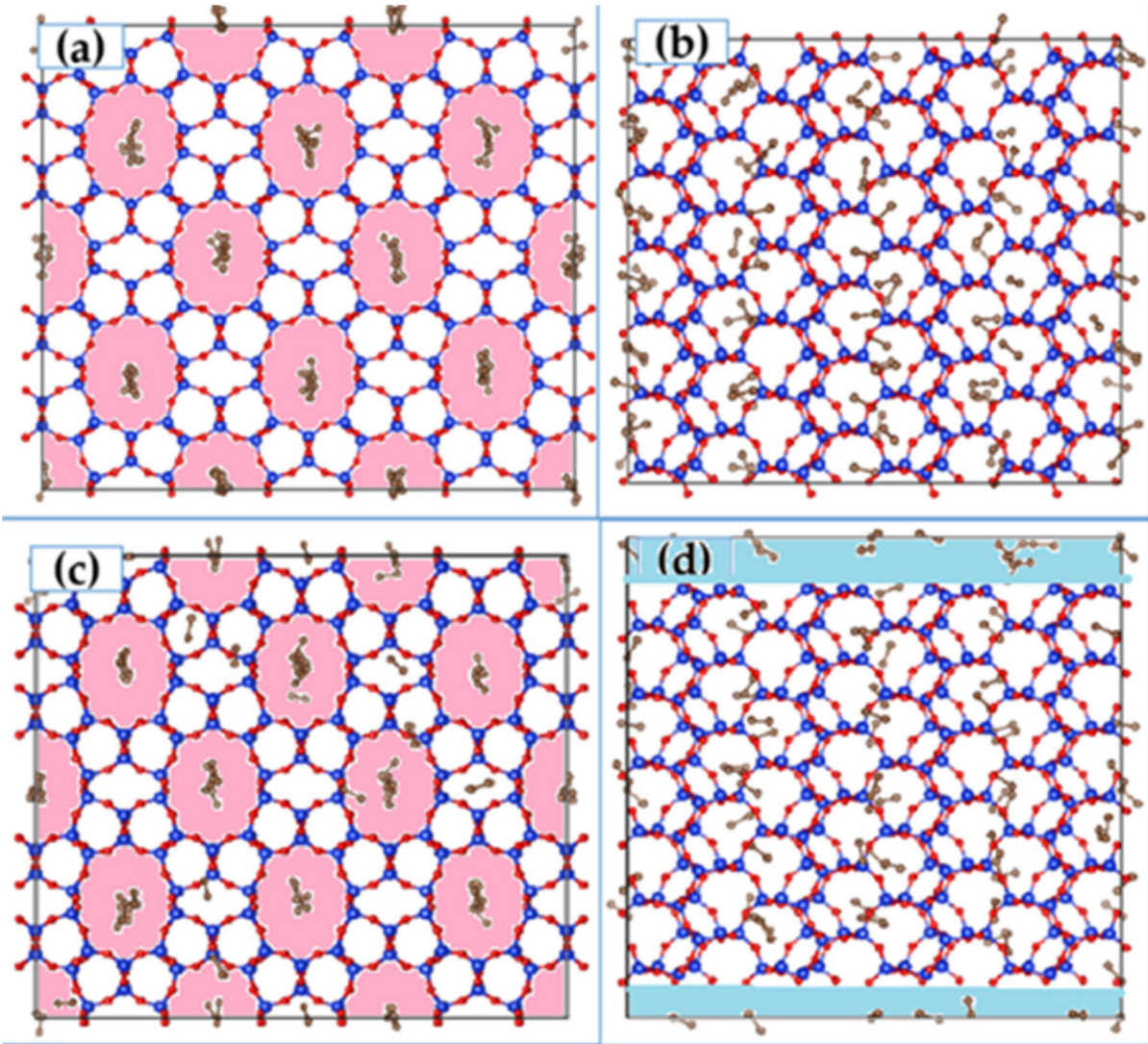

Figure 1. Snapshots from the simulation of ethane in ZSM-22 in $X-Y(\mathbf{a}, \mathbf{c})$ and $X-Z(\mathbf{b}, \mathbf{d})$ planes. $(\mathbf{a}, \mathbf{b})$ show the ZSM-22 simulation cell without any empty space inserted, while the simulation cell in (c,d) has empty space inserted in the Z-direction. Elliptical channels running in the Z-direction can be seen in the $X-Y$ plane (a,c) (regions colored in light pink) whereas the empty space inserted in the Z-direction can be seen in (d) (slab-like regions highlighted in light blue). Blue, red, and gray spheres, respectively, represent $\mathrm{Si}, \mathrm{O}$, and $\mathrm{CH}_{3}$ pseudo-atoms.

The interactions between the adsorbate molecules and those between the zeolites and the adsorbates were modeled in terms of the Lennard-Jones ( $\mathrm{LJ}$ ) potential in addition to the Coulombic interactions between entities with partial electrostatic charges. In general, the intermolecular force field took the following form:

$$
U_{i j}=4 \varepsilon_{i j}\left[\left(\frac{\sigma_{i j}}{r_{i j}}\right)^{12}-\left(\frac{\sigma_{i j}}{r_{i j}}\right)^{6}\right]+\frac{q_{i} q_{j}}{4 \pi \epsilon_{0} r_{i j}}
$$

where $\varepsilon_{i j}$ is the depth of the potential well, $\sigma_{i j}$ is the distance at which the intermolecular potential between the atoms $i$ and $j$ becomes zero, the van der Waals radius, and $r_{i j}$ is the distance between atoms $i$, and $j, q_{i}$, and $q_{j}$ are the charges of the $i$ and $j$ atoms. TraPPEUA [19] force field was used to model the interaction between the guest molecules and united atom formalism was used to model ethane molecules with two $\mathrm{CH}_{3}$ pseudo-atoms connected to each other. Both $\mathrm{CO}_{2}$ as well as ethane were considered as rigid molecules with fixed bond lengths, $l\left(C-\mathrm{O}_{\mathrm{C}}\right)=0.116 \mathrm{~nm}\left(\mathrm{O}_{\mathrm{c}}\right.$ is used to denote the oxygen atom belonging to a $\mathrm{CO}_{2}$ molecule) and $l\left(\mathrm{CH}_{3}-\mathrm{CH}_{3}\right)=0.154 \mathrm{~nm}$. ZSM-22 was modeled using the CLAYFF force field [20]. All ZSM-22 atoms were kept fixed during all simulations. Values of the Lennard- 
Jones parameters corresponding to $\mathrm{CO}_{2}$ and ethane molecules have been summarized in Tables 1 and 2, respectively. The partial charges on the atoms of ZSM-22 and $\mathrm{CO}_{2}$ were taken as $q_{S i}=+2.1 e, q_{O}=-1.05 e, q_{c}=+0.7 e, q_{O c}=-0.35 e$. The force-field parameters used in this work have been used earlier for simulating the adsorption, structure, and dynamics of ethane and $\mathrm{CO}_{2}$ in silicalite-an all silica analogue of ZSM-5 $[14,15]$. The adsorption isotherms of both ethane and $\mathrm{CO}_{2}$ in silicalite obtained from a GCMC simulation [15] using these parameters agreed well with the experimental isotherms obtained by Sun et al. [21] (see Figure 7 in ref. [15]).

Table 1. Lennard-Jones parameters for the $\mathrm{CO}_{2}-\mathrm{ZSM}-22$ interactions.

\begin{tabular}{cccccccc}
\hline $\begin{array}{c}\text { Interacting Molecules } \\
\text { LJ Parameters }\end{array}$ & $\mathrm{Si}-\mathrm{C}$ & $\mathrm{Si}-\mathrm{O}_{\mathbf{C}}$ & $\mathrm{O}-\mathrm{C}$ & $\mathrm{O}_{\mathrm{O}} \mathrm{O}_{\mathbf{C}}$ & $\mathrm{C}-\mathrm{C}$ & $\mathrm{C}-\mathrm{O}_{\mathbf{C}}$ & $\mathrm{O}_{\mathbf{C}}-\mathrm{O}_{\mathbf{C}}$ \\
\hline$\varepsilon_{i j} \mathrm{KJ} / \mathrm{mol}$ & 0.00131 & 0.00225 & 0.38163 & 0.65359 & 0.224 & 0.38362 & 0.657 \\
$\sigma_{i j} \AA$ & 3.051 & 3.176 & 2.983 & 3.108 & 2.8 & 2.925 & 3.05 \\
\hline
\end{tabular}

Table 2. Lennard-Jones parameters for ethane-ZSM-22 interactions.

\begin{tabular}{cccc}
\hline $\begin{array}{c}\text { Interacting Molecules } \\
\text { LJ Parameters }\end{array}$ & $\mathbf{S i}-\mathrm{CH}_{\mathbf{3}}$ & $\mathbf{O}-\mathrm{CH}_{\mathbf{3}}$ & $\mathbf{C H}_{\mathbf{3}}-\mathbf{C H}_{\mathbf{3}}$ \\
\hline$\varepsilon_{i j} \mathrm{KJ} / \mathrm{mol}$ & 0.00251 & 0.72795 & 0.815 \\
$\sigma_{i j} \AA$ & 3.5264 & 3.458 & 3.750 \\
\hline
\end{tabular}

Simulations were performed using the DL-POLY classic molecular dynamics simulation package [22]. All simulations were carried out in the NVT ensemble at a temperature of $300 \mathrm{~K}$. The Nose-Hoover thermostat was used to regulate the temperature with a relaxation time of $1 \mathrm{ps}$. Simulations were run for a total of $2 \mathrm{~ns}$ and a calculation time step of $1 \mathrm{fs}$ was used for the simulations. Following the TraPPE-UA convention, a cut-off distance of $14 \AA$ was used. In order to ensure equilibrium of the system, an equilibration time of $0.5 \mathrm{~ns}$ was used before the production process. Equilibration of the system was confirmed by inspecting the evolution of total energy and temperature of the system which exhibited stable values within acceptable fluctuation limits after $0.5 \mathrm{~ns}$. During the production process of $1.5 \mathrm{~ns}$, instantaneous positions and velocities of all the atoms/pseudo-atoms were recorded after every $20 \mathrm{fs}$.

\section{Results}

\subsection{Structure}

\subsubsection{Distribution of the Guest Molecules in a Channel}

Figure 2 shows the distribution of the guest molecules in a randomly selected pore of ZSM-22. In this figure, we present the logarithm of the instances when a guest molecule was found at a given location during the entire production time of $1.5 \mathrm{~ns}$. The distribution for $\mathrm{CO}_{2}$ is shown on the left, while that for ethane is shown on the right as the loadings increase from bottom to top. Elongated regions of non-zero intensity emulate the elliptically shaped pores that are slightly elongated along the Y-direction (see Figure 1a,c). At the lowest loadings, $\mathrm{CO}_{2}$ prefers two locations that are close to the pore wall and roughly symmetrically distributed about the pore axis (two regions of high intensity). As the loading is increased, the separation between these regions becomes somewhat diffused. Ethane molecules on the other hand prefer to reside at the center of the pore (a single high-intensity region located at the center) and exhibit no significant loading dependence of their distribution. 

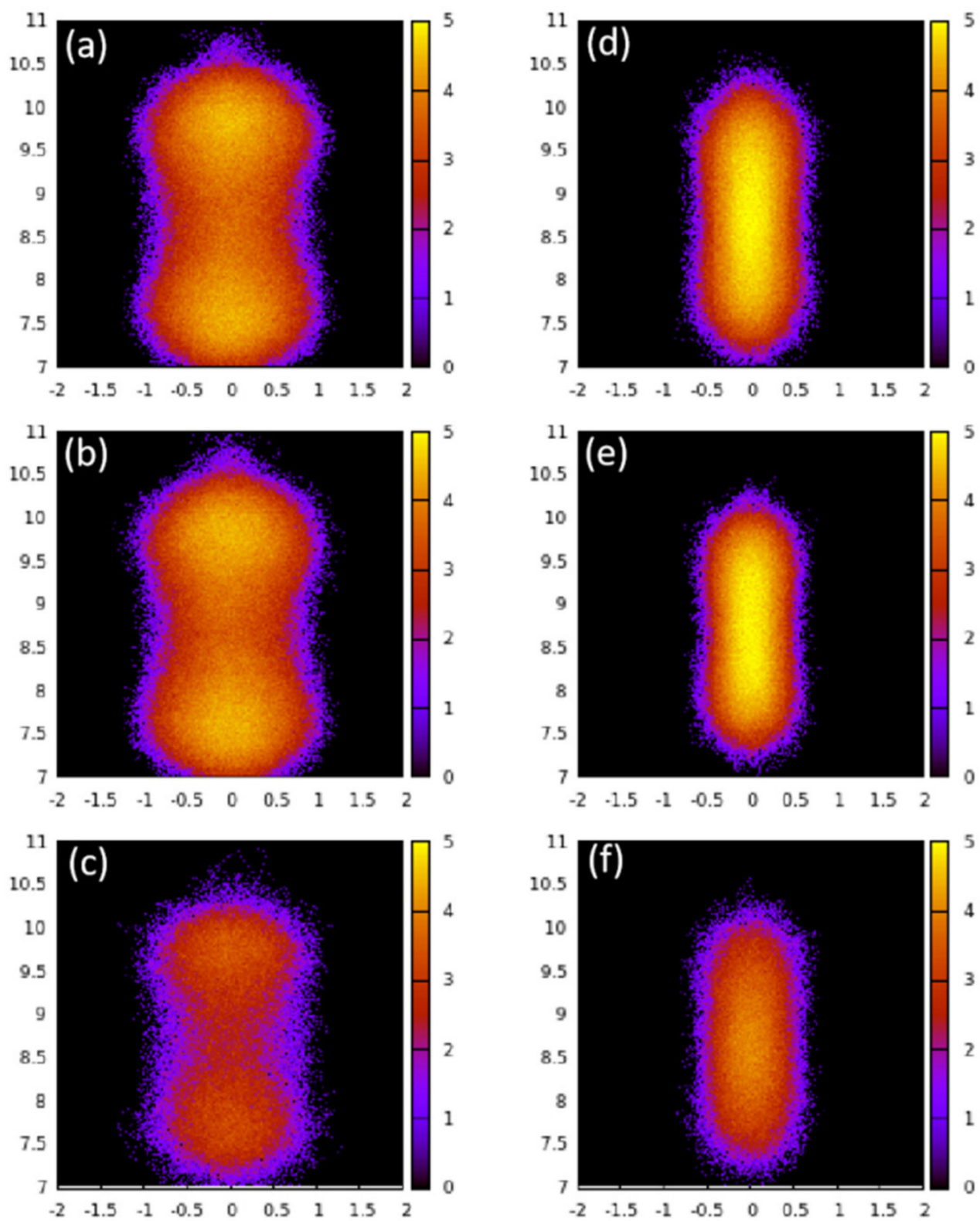

Figure 2. Distribution of guest molecules $\mathrm{CO}_{2}(\mathbf{a}-\mathbf{c})$ and ethane $(\mathbf{d}-\mathbf{f})$ in a pore of ZSM-22 at the guest loadings of $72(\mathbf{a}, \mathbf{d}), 52(\mathbf{b}, \mathbf{e})$, and $24(\mathbf{c}, \mathbf{f})$ molecules in the simulation cell. The intensity represents the logarithm of the number of times a guest molecule is found at a position with the given coordinates.

\subsubsection{Orientational Structure of the Guest Molecules}

In Figure 3, the orientational distribution of the guest molecules are presented in terms of the angle made by the molecular axis with respect to the different Cartesian directions noted. The plots also show the isotropic distribution expected for a system that exhibits no preferred orientation (black curves). Any deviation from this isotropic distribution indicates orientational ordering where the extent of the deviation is proportional to the degree of orientational ordering. A high degree of orientational order is exhibited by both ethane and $\mathrm{CO}_{2}$ adsorbed in the pores of ZSM-22. The order for $\mathrm{CO}_{2}$ is approximately independent of loading, whereas higher loading leads to some disorder in the orientational distribution for ethane. 

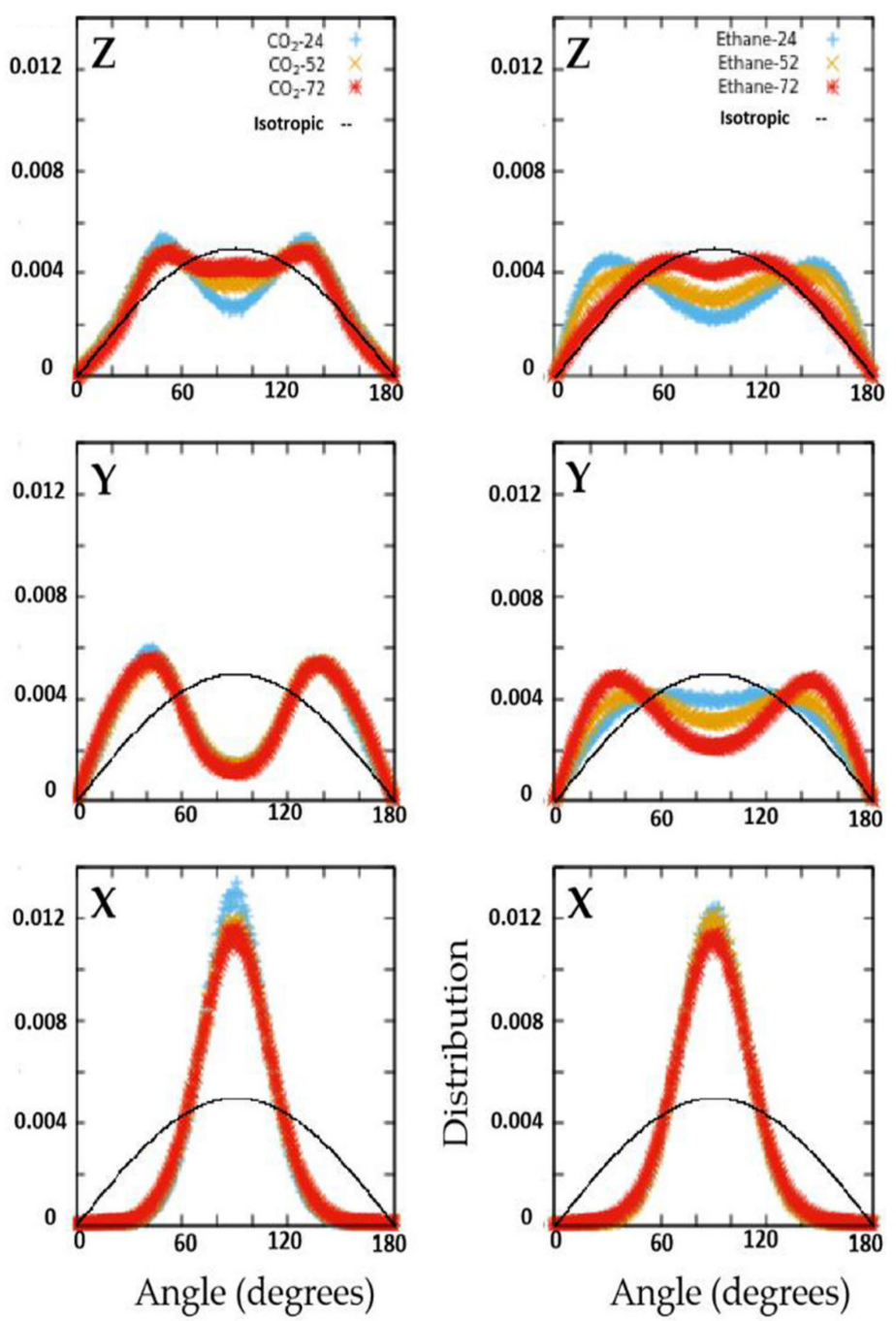

Figure 3. Distribution of angles made by the molecular axis of the guest molecules with respect to Cartesian directions for all loadings investigated in the case of ethane and $\mathrm{CO}_{2}$. The distribution for an isotropic case with no preferred orientation is shown as a black curve for reference.

\subsection{Dynamics}

\subsubsection{Translational Motion}

In Figure 4, the mean squared displacement is shown for different guest molecules at the intermediate loading of 52 molecules. While $\mathrm{CO}_{2}$ exhibits a lower value of mean squared displacement (MSD) at all times, when the electrostatic interactions are turned off, the resulting MSD values (shown as $\mathrm{nCO}_{2}$ curve in the plot) are higher than that for ethane. In the right panel of this figure, the MSD vs. time plot is shown in the log-log plot that helps reveal the behavior of motion as a function of time. This plot exhibits three distinct regions, with separating boundaries marked with arrows. The initial region of rapid increase in MSD lasts for less than 1 ps and represents the ballistic regime of motion. A second region that lasts for a few tens of picosecond shows a relatively slower increase of MSD with time, after which the increase is faster in the third region. The second region represents a restricted sub-diffusion motion, while the third region is the region of diffusive motion. Self-diffusion coefficient $\mathrm{D}_{\mathrm{s}}$ can be obtained using the Einstein relation by fitting the diffusive region of MSD vs. time curve by a straight line, the slope of which is proportional to $D_{s}$. Values of $D_{s}$ obtained thus for the various systems investigated are reported in Table 3. 

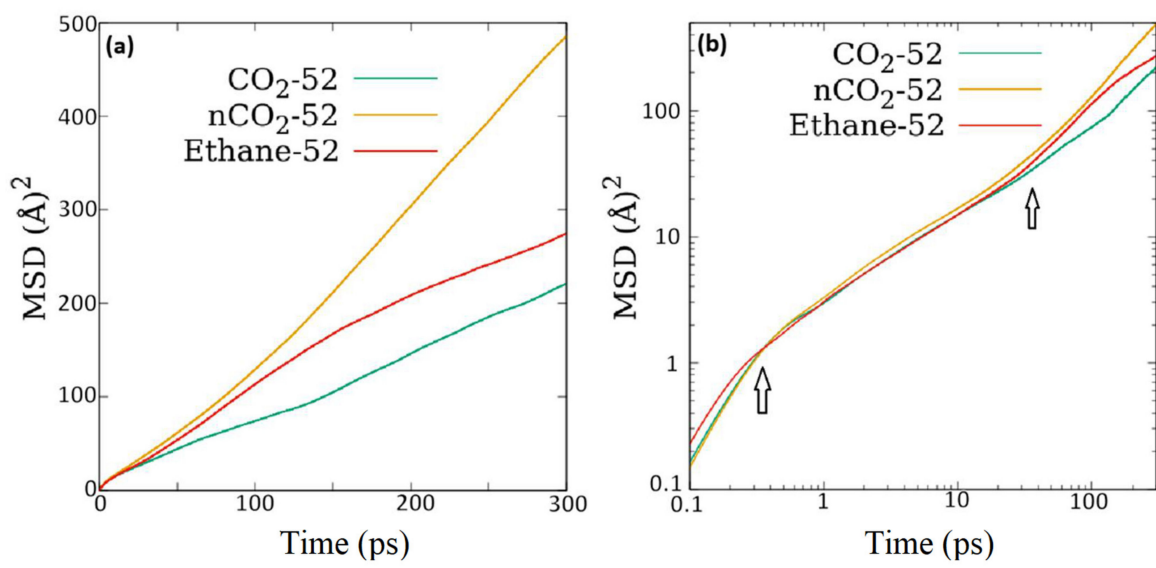

Figure 4. Mean squared displacement (MSD) as a function of time in (a) linear scale, and (b) log-log scale. Three distinct regions corresponding to ballistic, sub-diffusive, and diffusive motions can be identified in (b). $\mathrm{nCO}_{2}$ refers to $\mathrm{CO}_{2}$ with no electrostatic interactions used in the simulation.

Table 3. Self-diffusion coefficients in the units of $10^{-10} \mathrm{~m}^{2} / \mathrm{s}$ obtained from the long-time behavior of MSD from different simulations.

\begin{tabular}{cccc}
\hline System & 72 Molecules & 52 Molecules & 24 Molecules \\
\hline Ethane in ZSM-22 & $5.55 \pm 0.01$ & $17.47 \pm 0.01$ & $54.06 \pm 0.02$ \\
\hline $\mathrm{CO}_{2}$ in ZSM-22 & $5.18 \pm 0.01$ & $11.43 \pm 0.01$ & $32.61 \pm 0.52$ \\
\hline $\begin{array}{c}\text { Chargeless } \mathrm{CO}_{2} \text { in ZSM-22 } \\
\begin{array}{c}\text { Ethane in ZSM-22 with } \\
\text { pore connecting space }\end{array}\end{array}$ & $-33.91 \pm 0.01$ & $30.14 \pm 0.01$ & - \\
\hline $\begin{array}{c}\mathrm{CO}_{2} \text { in ZSM-22 with pore } \\
\text { connecting space }\end{array}$ & $1.56 \pm 0.01$ & - & - \\
\hline
\end{tabular}

For a nanoporous system with an anisotropic pore network, the diffusivity of the guest molecules can be expected to also exhibit anisotropy. The MSD components calculated along different directions are shown in Figure 5 for the three systems at the intermediate loading of 52 molecules. As expected, the MSD in the directions perpendicular to the direction of the pore-axis is heavily suppressed in all cases. At longer times where the diffusive motion starts, the MSD along the direction of the pore-axis (i.e., Z-direction) is identical to the overall MSD.
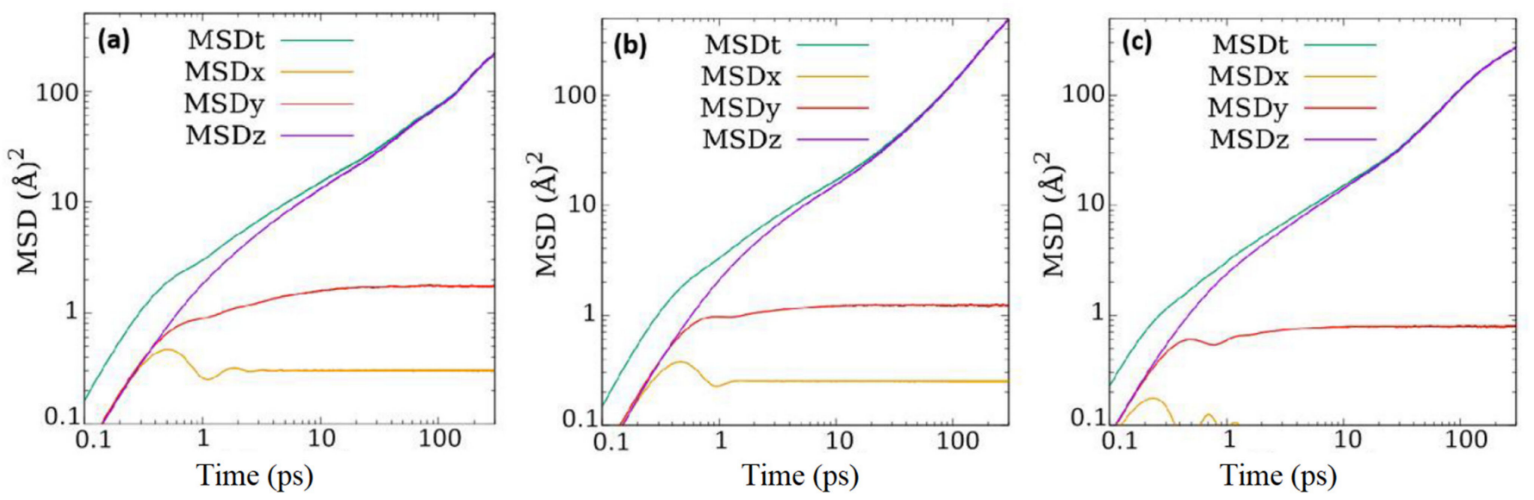

Figure 5. Components of mean squared displacement (MSD) along different directions and the overall MSD (MSDt) for (a) $\mathrm{CO}_{2}$, (b) $\mathrm{CO}_{2}$ without electrostatic interactions, and (c) ethane in ZSM-22 at a loading of 52 molecules in the simulation cell. 


\subsubsection{Reorientational Motion}

Rotational or reorientational motion of the guest molecules was investigated by following the orientation of the molecular axis over time. In particular, we calculated the correlation function $\mathrm{R}(\mathrm{t})=\langle\boldsymbol{u}(0) \cdot \boldsymbol{u}(\mathrm{t})>$ of a unit vector $\boldsymbol{u}$ attached to the molecular axis. The $R(t)$ for the representative systems investigated is shown in Figure 6. The $R(t)$ vs. time curves can be divided into two distinct regions separated by a wobble at around 1 ps. The second region represents the long-time overall rotation of the guest molecules. To obtain the time scales of rotational motion, we fitted the second region of $R(t)$ with an exponential function $\mathrm{R}(\mathrm{t})=a \exp (-\mathrm{t} / \tau)+c$ with $a, \tau$, and $c$ as fitting parameters. The time constants $\tau$ obtained thus are listed in Table 4 .

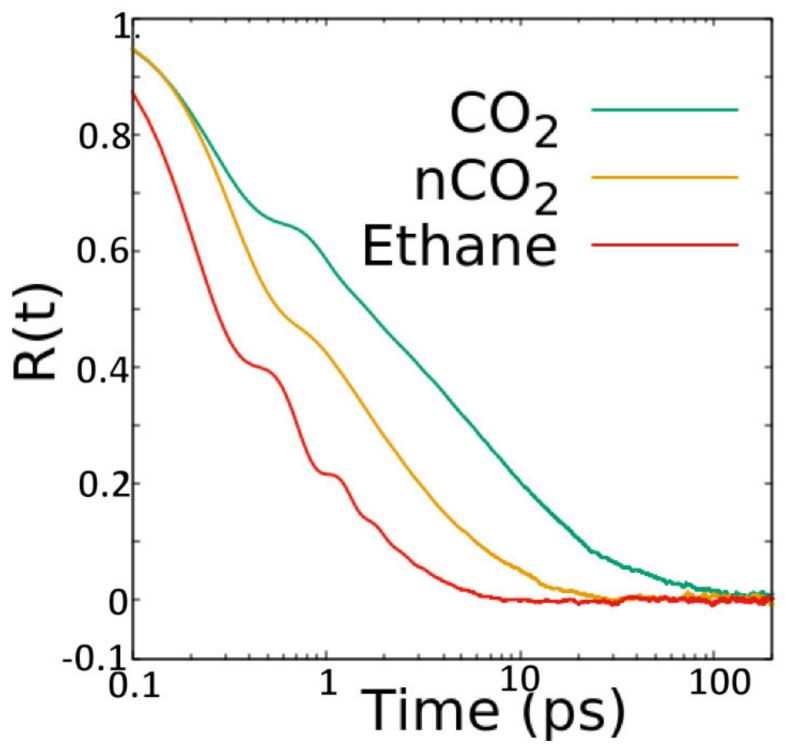

Figure 6. Reorientational correlation function $\mathrm{R}(\mathrm{t})$ for $\mathrm{CO}_{2}$ and ethane in $\mathrm{ZSM}-22$ at a loading of 52 molecules in the simulation cell. In the legend, $\mathrm{nCO}_{2}$ indicates results from the simulation of $\mathrm{CO}_{2}$ without electrostatic interactions.

Table 4. Time constants $\tau$ (in ps) of the decay of the rotational correlation functions above 1 ps.

\begin{tabular}{cccc}
\hline System & 72 Molecules & 52 Molecules & 24 Molecules \\
\hline Ethane in ZSM-22 & $1.85 \pm 0.01$ & $1.76 \pm 0.01$ & $1.89 \pm 0.02$ \\
\hline $\mathrm{CO}_{2}$ in ZSM-22 & $13.3 \pm 0.02$ & $24.71 \pm 0.11$ & $42.2 \pm 0.24$ \\
\hline $\begin{array}{c}\text { Chargeless } \mathrm{CO}_{2} \text { in ZSM-22 } \\
\begin{array}{c}\text { Ethane in ZSM-22 with } \\
\text { pore connecting space }\end{array}\end{array}$ & $-2.16 \pm 0.01$ & $2.87 \pm 0.02$ & - \\
\hline $\begin{array}{c}\mathrm{CO}_{2} \text { in ZSM-22 with pore } \\
\text { connecting space }\end{array}$ & $46.13 \pm 0.01$ & - & - \\
\hline
\end{tabular}

\subsection{Effect of Connecting the Pores Artificially by Inserting Inter-Crystalline Space}

ZSM-22 has parallel channel-like pores running in the Z-direction that are isolated from each other. The effects of inter-connecting these pores was studied by inserting extra empty space in the simulation on top and bottom of the simulation cell. These empty spaces provide a transit for the molecules in one pore to migrate to another pore. The effect of this inter-connection on the MSD and $\mathrm{R}(\mathrm{t})$ of both $\mathrm{CO}_{2}$ and ethane are shown in Figure 7 (left and right panels respectively). The effect of this pore connectivity via inter-crystalline spacing is different for the two guest molecules. While both translational and rotational motion of $\mathrm{CO}_{2}$ are suppressed on connecting the pores, ethane represents an enhancement of translational motion but a suppression of rotational motion when the pores are connected 
via the inter-crystalline space. The last of these effects, i.e., suppression of rotational motion of ethane in connected pores is evident in Figure 7 (right panel) that exhibits a slower decay in the second region of $\mathrm{R}(\mathrm{t})$ for ethane in the connected pores as compared to that for isolated pores.
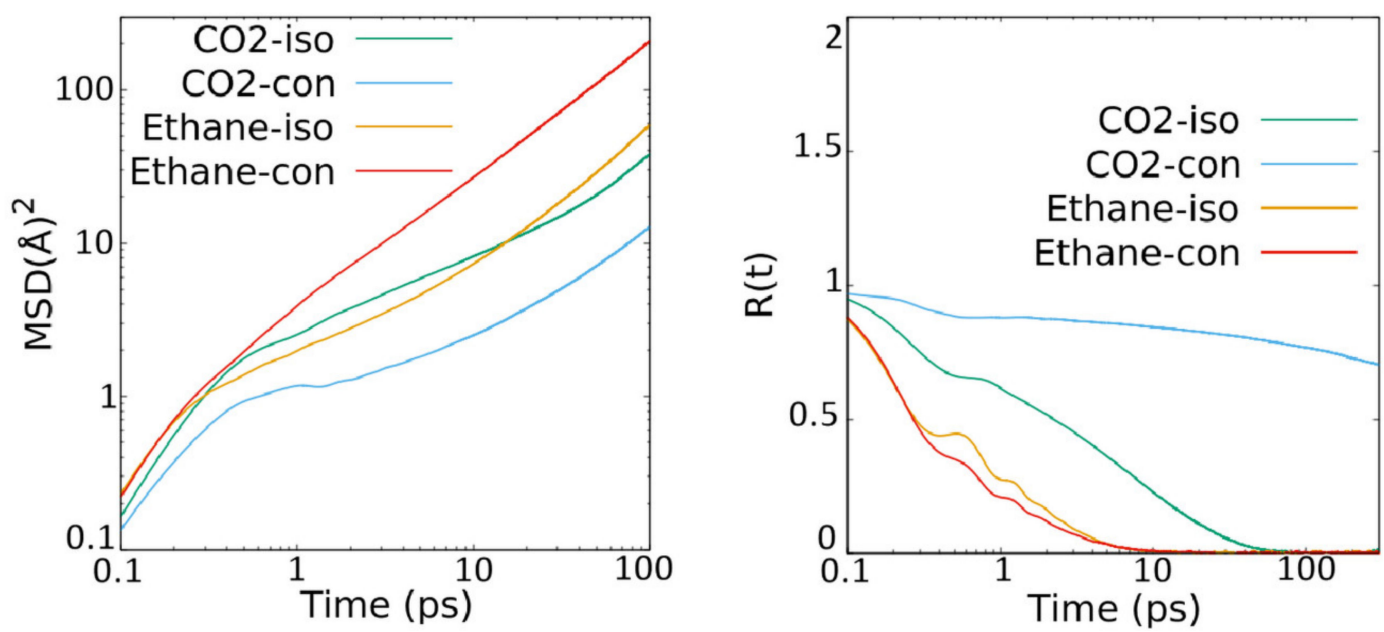

Figure 7. MSD (left) and $\mathrm{R}(\mathrm{t})$ (right) of ethane and $\mathrm{CO}_{2}$ in $\mathrm{ZSM}-22$ at a loading of 72 molecules in the simulation cell. In the legend, the suffices "iso" and "con" are used to distinguish between the simulations involving unmodified ZSM-22 with isolated pores and ZSM-22 modified by inserting inter-crystalline space that results in the pores being connected via the inter-crystalline space.

\section{Discussion}

The structural and dynamical behavior of both fluids mimic the structure of the pores. As seen in Figure 2, the fluid molecules tend to occupy the pores in positions that are elongated along the $\mathrm{Y}$ axis, in consistency with the pores that are slightly elongated along the $Y$ axis (see Figure 1). This slight elongation along the $Y$ axis also gives rise to preference to orient perpendicular to the $X$-axis (see Figure 3). While $\mathrm{CO}_{2}$ molecules prefer to lie making an angle of roughly 45 degrees with respect to both $Y$ - and Z-directions, the ethane molecules show a preference to make smaller (or larger) angles with Z (or Y-directions) at lower loadings, progressively shifting towards larger (or smaller) angles with Z (or Ydirections). Thus, while ethane exhibits a range of orientations with respect to the pore axis (and hence pore wall) depending on the loading, $\mathrm{CO}_{2}$ molecules show a preference to lie making an angle of 45 degrees with the pore surface irrespective of the loading. A preference for this orientation with respect to the substrate has been observed for $\mathrm{CO}_{2}$ in several other systems $[15,23]$.

The results presented here show that the electrostatic interactions play an important role in the behavior of $\mathrm{CO}_{2}$ under confinement. This has been reported earlier in several other studies $[15,24]$. The quadrupole moment of $\mathrm{CO}_{2}$ is responsible for selective adsorption of $\mathrm{CO}_{2}$ on silica surfaces, leading to reduced mobility. This restriction on the motion of $\mathrm{CO}_{2}$ due to the electrostatic interactions is relatively weaker as compared to the restriction imposed on slightly larger polar molecules like acetonitrile and acetaldehyde [6] that are rendered practically immobile in the channels of ZSM-5 of similar dimensions as in ZSM-22 investigated here.

A quick comparison of the overall self-diffusion coefficients reported in Table 3 here with those for the two fluids reported in ZSM-5 [14] suggests that although the diffusivity of ethane in ZSM-22 is same as that in ZSM-5, that of $\mathrm{CO}_{2}$ is slightly reduced in ZSM22 compared to that in ZSM-5. However, there are some important caveats to consider. As stated in the introduction, the pore network of ZSM-22 is similar in dimensions to that in ZSM-5 but without inter-connections between different channels. The straight channellike pores in ZSM-22 are aligned along the crystallographic axis $c$ and are analogous to 
the straight channels in ZSM-5 that run parallel to $b$ axis. One direct consequence of the difference in the pore structure in ZSM-22 and ZSM-5 is that while in the former, the mean squared displacement at longer time is entirely along the direction of straight channels, that in the latter is distributed along all directions even though the component along the direction of straight channels is dominant. This is because of the existence of sinusoidal channels that run perpendicular to the straight channels in ZSM-5, giving rise to motion along the plane perpendicular to the straight channels. These sinusoidal channels also provide a connection between the straight channels. The effect of the presence of this connection between the pores in ZSM-5 or their absence in ZSM-22 can be understood by comparing the one-dimensional diffusivity of fluid molecules along the pore direction, i.e., in the Y-direction in ZSM-5 and in Z-direction in ZSM-22. For CO 2 loaded in ZSM-5 at partial gas pressures between 0.1 to 100 bar, this diffusivity was in the range of 66 to $12 \times 10^{-10} \mathrm{~m}^{2} / \mathrm{s}$, while for ethane in ZSM-5, it was in the range 71 to $5.5 \times 10^{-10} \mathrm{~m}^{2} / \mathrm{s}$ for these partial pressures [14]. The different loadings as reported here also correspond roughly to the same range of partial pressures. As the MSD along the pore axis in ZSM-22 is identical to the overall MSD at long times, the overall self-diffusion coefficients listed in Table 3 can be used as a measure of the diffusion coefficient along the pore axis. However, it should be noted that the self-diffusion coefficients along the pore axis calculated for ZSM-5 as mentioned above were obtained by considering one-dimensional motion while those reported in Table 3 for ZSM-22 are obtained considering 3-D motion. Thus, a fair comparison between the two sets of diffusion coefficients (those for ZSM-5 and ZSM22) requires multiplying the values listed in Table 3 by a factor of 3 (The self-diffusion coefficient is obtained via Einstein relation as $D_{s}=\operatorname{MSD} /\left(2 n_{d} t\right)$, where $n_{d}$ is the number of dimensions; $\mathrm{n}_{\mathrm{d}}=3$ for the overall $\mathrm{D}_{\mathrm{s}}$ as listed in Table $3 ; \mathrm{n}_{\mathrm{d}}=1$ for one-dimensional diffusion. Thus, to convert the $3-\mathrm{D} \mathrm{D}_{\mathrm{s}}$ values listed in Table 3 to a 1-D self-diffusion coefficient, the former needs to be multiplied by 3 ). With this normalization, we obtain the one-dimensional self-diffusion coefficients of $\mathrm{CO}_{2}$ and ethane in the direction of the pore axis of ZSM-22 in the range of 97 to $15.6 \times 10^{-10}$ and 162 to $16.6 \times 10^{-10} \mathrm{~m}^{2} / \mathrm{s}$, respectively. These self-diffusion coefficients along the pore axis in ZSM-22 are thus higher than those in ZSM-5, suggesting that pore connectivity hinders the diffusivity. This could be because of the collisions with the cross-running molecules in the sinusoidal channels that slow down the motion of molecules in the straight channels of ZSM-5, while in absence of pore connectivity, the molecules in the channel-like pores of ZSM-22 encounter no such collisions and hence mov without any hindrance. It has been found that $\mathrm{CO}_{2}$ molecules show a preference for partitioning in the intersections of the straight and sinusoidal channels in ZSM-5, whereas ethane molecules are more likely to be found in the sinusoidal channels at high loadings [14]. However, in another study [25] it was found that the residence auto-correlation function of ethane in the intersections of ZSM- 5 decayed slower than those for ethane in straight and sinusoidal channels by a factor of more than 2 . This means that even though the energetics favor the partitioning of ethane in sinusoidal channels, once they reach the intersections, they are likely to spend a significant amount of time there, probably undergoing a rattling motion. This rattling motion of the guest molecules in the intersection provides a hindrance to the motion of other guest molecules through the straight channels.

While the translational motion of both ethane and $\mathrm{CO}_{2}$ is facilitated by the absence of pore connectivity, the effect of pore connectivity on the rotational motion is different on the two fluids. For ethane, the time scales of rotational motion as listed in Table 4 (between 1.85 and 1.89 ps) are smaller than those for ethane in ZSM-5 (between 2 and 12 ps) [14], suggesting a faster rotation for ethane in ZSM-22. In contrast to this, the rotational time scales for $\mathrm{CO}_{2}$ in ZSM-22 (between 13 and $42 \mathrm{ps)} \mathrm{are} \mathrm{larger} \mathrm{than} \mathrm{those} \mathrm{in} \mathrm{ZSM-5} \mathrm{(between}$ 7 and 22 ps) [14], suggesting a slower rotation of $\mathrm{CO}_{2}$ in ZSM-22. Rotational motion of both guest molecules in ZSM-22 is enhanced at higher loadings. A similar enhancement of rotational motion at higher loading was also observed for ethane and $\mathrm{CO}_{2}$ in ZSM-5 [14,25] and was explained in terms of a decreased orientational order at higher loadings. 
In the absence of inter-connections between the pores of ZSM-22, we added artificial inter-connections by exposing the pores to common inter-crystalline space. The artificial addition of such inter-crystalline space has been earlier used to emulate the powder samples in simulations [15,26-28]. In the present case, this inter-crystalline space effectively provided a connection between the straight channel-like pores of ZSM-22. Comparing the values of overall self-diffusion coefficients in ZSM-22 with and without pores connected via inter-crystalline space listed in Table 3, it can be seen that the effects of this artificially imposed pore connectivity is different on the two fluids. While ethane diffusion is facilitated by pore connectivity, that of $\mathrm{CO}_{2}$ is suppressed significantly. However, we note that as the inter-crystalline space added for connecting the pores provides a three-dimensional slab-like space in which the molecules are free to move in X-and Y-directions in addition to the Z-direction, a fair comparison with the case of isolated pores in ZSM-22 where motion occurs only along the Z-direction would require limiting the comparison to diffusivity along the Z-direction. Self-diffusion coefficients of ethane and $\mathrm{CO}_{2}$ along the Z-direction obtained for ZSM-22 modified with inter-crystalline space are, respectively, $19.92 \times 10^{-10}$ and $2.01 \times 10^{-10} \mathrm{~m}^{2} / \mathrm{s}$. Compared to the values of $16.65 \times 10^{-10}$ and $15.54 \times 10^{-10} \mathrm{~m}^{2} / \mathrm{s}$ for the self-diffusion coefficient along Z-direction of ethane and $\mathrm{CO}_{2}$ in $\mathrm{ZSM}-22$ with isolated pores, (obtained by normalizing the values listed in Table 3 for one-dimensional motion), we see that the effect of pore connectivity via inter-crystalline space is indeed different for the two fluids-enhancement of diffusivity for ethane and its suppression for $\mathrm{CO}_{2}$ in ZSM-22. Unlike the difference in the effects of pore connectivity via inter-crystalline space on translational motion of ethane and $\mathrm{CO}_{2}$, the rotational motion of both ethane and $\mathrm{CO}_{2}$ were found to be suppressed by inter-crystalline space (Table 4).

In Figure 8 we show a snapshot of $\mathrm{CO}_{2}$ adsorbed in ZSM-22 with inter-crystalline space inserted at the bottom and top of the simulation cell along the Z-direction. The corresponding snapshot for ethane in this system is shown in Figure 1d. A clear distinction can be seen between the behavior of ethane and $\mathrm{CO}_{2}$ in this system by comparing Figures 8 and $1 \mathrm{~d}$. While ethane molecules prefer to be adsorbed in the ZSM-22 pores, more $\mathrm{CO}_{2}$ molecules can be seen adsorbed on the surface of the crystallite in the inter-crystalline space, suggesting a preference for the surface adsorption compared with adsorption in the crystalline pores. This is consistent with the difference observed between ethane and $\mathrm{CO}_{2}$ adsorption in the inter-crystalline space of ZSM-5 [15]. The curvature of the silica surface plays an important role in the preferential adsorption of $\mathrm{CO}_{2}$ on the surface vis-a-vis the pores. While the silica surface in the vicinity of the inter-crystalline space is flat, that in the pores has a strong curvature such that a $\mathrm{CO}_{2}$ molecule is wrapped around by it. As a result, the silica atoms on the opposite sides compete for and nullify their interaction with $\mathrm{CO}_{2}$, thereby freeing the $\mathrm{CO}_{2}$ molecule to move along the pore axis. This nullification of strong surface interactions by opposite pore surfaces has been found to enhance the diffusivity of guest molecules in zeolite pores and is termed as the "levitation effect" [29]. Strong adsorption of $\mathrm{CO}_{2}$ on the crystallite surface has important consequences for the dynamical behavior. Connecting the pores of ZSM-22 by inserting inter-crystalline space leads to a suppression of both translational as well as rotational dynamics of $\mathrm{CO}_{2}$, while with weaker substrate-fluid interactions in the case of ethane, the inserted space provides greater freedom to ethane to move between the pores, thus enhancing its mobility. Further, the intersection between the channels and the interconnecting slab-like space is open and the guest molecules show no preference to localize at these intersections, in contrast to the case of ZSM-5. This reduces the probability of collisions considerably and thereby in absence of collisions, the guest molecules in the straight channels of ZSM-22 are free to move in absence of any hindrance. This suggests that the effects of interconnecting the pores depend upon the way the pores are connected. 


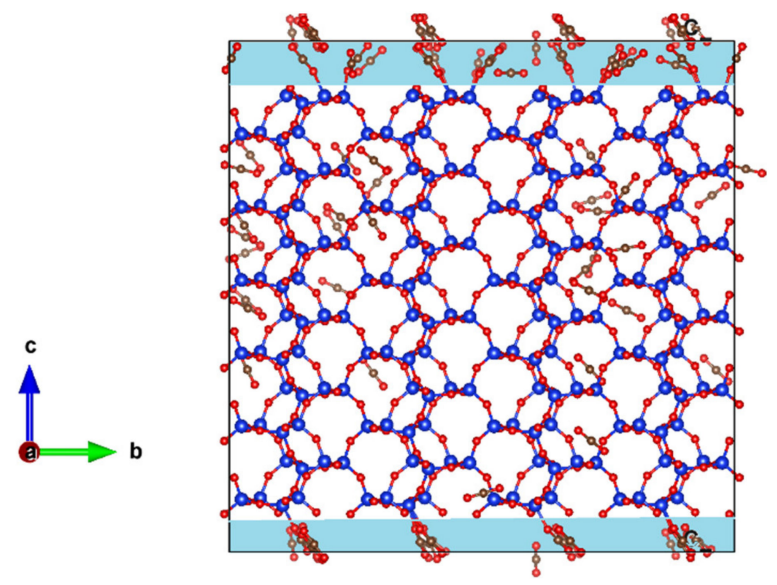

Figure 8. Snapshots in $\mathrm{X}-\mathrm{Z}$ plane from the simulation of $\mathrm{CO}_{2}$ in $\mathrm{ZSM}-22$ with empty space inserted in the Z-direction. Blue, red, and gray spheres respectively represent $\mathrm{Si}, \mathrm{O}$, and $\mathrm{C}$ atoms. $\mathrm{More} \mathrm{CO}_{2}$ molecules can be seen strongly adsorbed on the surface of ZSM-22 in the inter-crystalline space (shown in light blue) as compared to inside the pores.

\section{Conclusions}

MD simulations of ethane and $\mathrm{CO}_{2}$ reported here, in combination with a previous study involving ZSM-5, help us understand the role of pore connectivity in determining the structural and dynamical behavior of the confined fluids. We found that the effects of pore connectivity depend not only on the type of the fluid under confinement but also the way the pore connectivity is imposed. While the translational motion of both ethane and $\mathrm{CO}_{2}$ in ZSM-22 is suppressed as a result of connecting the pores by perpendicular quasi one-dimensional pores of similar dimensions, the effect of connecting the pores by inserting a slab-like 2-dimensional inter-crystalline space is different on the translational motion of the two fluids. For ethane, pores connected via inter-crystalline space facilitate translational motion but suppress rotational motion, whereas in the case of $\mathrm{CO}_{2}$, both types of motion are suppressed by pore connection due to the strong interaction of $\mathrm{CO}_{2}$ with the surface of the substrate that reduces its mobility. This computational study is a first step toward understanding the interplay of fluid-substrate interactions, pore connectivity, and pore dimensionality. The effects of pore connectivity involve a variety of other factors including the degrees of connectivity, pore volume, and surface areas, which are not addressed in this work but will be addressed in future works.

Author Contributions: Conceptualization, S.G.; methodology, S.G.; validation, S.G.; formal analysis, M.M.K. and S.G.; investigation, M.M.K. and S.G.; writing-original draft preparation, M.M.K. and S.G.; writing-review and editing, M.M.K., S.G. and D.C.; funding acquisition, D.C. All authors have read and agreed to the published version of the manuscript.

Funding: This research was funded by the U.S. Department of Basic Energy, Office of Science, Office of Basic Energy Sciences, Division of Chemical Sciences, Geosciences and Biosciences, Geosciences Program, grant number DESC0006878 (D.C. and S.G.).

Institutional Review Board Statement: Not applicable.

Informed Consent Statement: Not applicable.

Data Availability Statement: The authors confirm that the data supporting the findings of this study are available within the article.

Acknowledgments: We would like to acknowledge STFC's Daresbury Laboratory for providing the package DL-Poly, which was used in this work. Figures in this manuscript were made using the freely available visualization and plotting softwares Vesta [18] (Figures 1 and 8) and Gnuplot [30] (Figures 2-7). 
Conflicts of Interest: The authors declare no conflict of interest. The funders had no role in the design of the study; in the collection, analyses, or interpretation of data; in the writing of the manuscript; or in the decision to publish the results.

\section{References}

1. Tomlison, A.A.G. Modern Zeolites: Structure and Function in Detergents and Petrochemicals (Materials Science Foundations, Vol 3); Trans Tech Publications: Zurich, Switzerland, 1998.

2. Gautam, S.S.; Ok, S.; Cole, D.R. Structure and Dynamics of Confined C-O-H Fluids Relevant to the Subsurface: Application of Magnetic Resonance, Neutron Scattering, and Molecular Dynamics Simulations. Front. Earth Sci. 2017, 5, 1-19. [CrossRef]

3. Van Koningsveld, H.; van Bekkum, H.; Jansen, J.C. On the location and disorder of the tetrapropylammonium (TPA) ion in zeolite ZSM-5 with improved framework accuracy. Acta Crystallogr. Sect. B 1987, 43, 127-132. [CrossRef]

4. Hahn, K.; Kärger, J.; Kukla, V. Single-File Diffusion Observation. Phys. Rev. Lett. 1996, 76, 2762-2765. [CrossRef] [PubMed]

5. Dhiman, I.; Bhowmik, D.; Shrestha, U.R.; Cole, D.R.; Gautam, S. Effect of molecular shape on rotation under severe confinement. Chem. Eng. Sci. 2018, 180, 33-41. [CrossRef]

6. Dhiman, I.; Shrestha, U.R.; Bhowmik, D.; Cole, D.R.; Gautam, S. Influence of molecular shape on self-diffusion under severe confinement: A molecular dynamics study. Chem. Phys. 2019, 516, 92-102. [CrossRef]

7. Gautam, S.; Liu, T.; Rother, G.; Jalarvo, N.; Mamontov, E.; Welch, S.; Sheets, J.; Droege, M.; Cole, D.R. Dynamics of Propane in Nanoporous Silica Aerogel: A Quasielastic Neutron Scattering Study. J. Phys. Chem. C 2015, 119, 18188-18195. [CrossRef]

8. Le, T.; Striolo, A.; Cole, D.R. $\mathrm{CO}_{2}-\mathrm{C}_{4} \mathrm{H}_{10}$ Mixtures Simulated in Silica Slit Pores: Relation between Structure and Dynamics. J. Phys. Chem. C 2015, 119, 15274-15284. [CrossRef]

9. Patankar, S.; Gautam, S.; Rother, G.; Podlesnyak, A.; Ehlers, G.; Liu, T.; Cole, D.R.; Tomasko, D.L. Role of Confinement on Adsorption and Dynamics of Ethane and an Ethane- $\mathrm{CO}_{2}$ Mixture in Mesoporous CPG Silica. J. Phys. Chem. C 2016, 120, 4843-4853. [CrossRef]

10. Elola, M.D.; Rodriguez, J. Preferential Adsorption in Ethane/Carbon Dioxide Fluid Mixtures Confined within Silica Nanopores. J. Phys. Chem. C 2019, 123, 30937-30948. [CrossRef]

11. Yan, J.; Zhang, B.; Wang, Z. Highly selective separation of $\mathrm{CO}_{2}, \mathrm{CH}_{4}$, and $\mathrm{C} 2-\mathrm{C} 4$ hydrocarbons in ultramicroporous semicycloaliphatic polyimides. ACS Appl. Mater. Interfaces 2018, 10, 26618-26627. [CrossRef]

12. Duan, J.; Higuchi, M.; Horike, S.; Foo, M.L.; Rao, K.P.; Inubushi, Y.; Fukushima, T.; Kitagawa, S. High $\mathrm{CO}_{2} / \mathrm{CH}_{4}$ and $\mathrm{C} 2$ hydrocarbons $/ \mathrm{CH}_{4}$ selectivity in a chemically robust porous coordination polymer. Adv. Funct. Mater. 2013, 23, 3525-3530. [CrossRef]

13. Chandra, D.; Vishal, V.; Bahadur, J.; Sen, D. A novel approach to identify accessible and inaccessible pores in gas shales using combined low-pressure sorption and SAXS/SANS analysis. Int. J. Coal Geol. 2020, 228, 103556. [CrossRef]

14. Gautam, S.; Liu, T.; Cole, D. Sorption, Structure and Dynamics of $\mathrm{CO}_{2}$ and Ethane in Silicalite at High Pressure: A Combined Monte Carlo and Molecular Dynamics Simulation Study. Molecules 2019, 24, 99. [CrossRef] [PubMed]

15. Gautam, S.; Cole, D.R. Effects of inter-crystalline space on the adsorption of ethane and $\mathrm{CO}_{2}$ in silicalite: Implications for enhanced adsorption. Phys. Chem. Chem. Phys. 2020, 22, 13951-13957. [CrossRef] [PubMed]

16. Kokotailo, G.T. The framework topology of ZSM-22: A high silica zeolite. Zeolites 1985, 5, 349-351. [CrossRef]

17. Yang, X.; Wu, M.; Qin, Z.; Wang, J.; Wen, T. Molecular dynamics simulations on single-file diffusions: Effects of channel potential periods and particle-particle interactions. J. Appl. Phys. 2009, 106, 084905. [CrossRef]

18. Momma, K.; Izumi, F. VESTA 3 for three-dimensional visualization of crystal, volumetric and morphology data. J. Appl. Crystallogr. 2011, 44, 1272-1276. [CrossRef]

19. Martin, M.G.; Siepmann, J.I. Transferable Potentials for Phase Equilibria. 1. United-Atom Description of n-Alkanes. J. Phys. Chem. B 1998, 102, 2569-2577. [CrossRef]

20. Cygan, R.T.; Liang, J.-J.; Kalinichev, A.G. Molecular Models of Hydroxide, Oxyhydroxide, and Clay Phases and the Development of a General Force Field. J. Phys. Chem. B 2004, 108, 1255-1266. [CrossRef]

21. Sun, M.S.; Shah, D.B.; Xu, H.H.; Talu, O. Adsorption Equilibria of C1 to C4 Alkanes, $\mathrm{CO}_{2}$, and SF 6 on Silicalite. J. Phys. Chem. B 1998, 102, 1466-1473. [CrossRef]

22. Todorov, I.T.; Smith, W.; Trachenko, K.; Dove, M.T. DL_POLY_3: New dimensions in molecular dynamics simulations via massive parallelism. J. Mater. Chem. 2006, 16, 1911-1918. [CrossRef]

23. Liu, T.; Gautam, S.; Cole, D.R.; Patankar, S.; Tomasko, D.; Zhou, W.; Rother, G. Structure and dynamics of ethane confined in silica nanopores in the presence of $\mathrm{CO}_{2}$. J. Chem. Phys. 2020, 152, 084707. [CrossRef]

24. Gowers, R.J.; Farmahini, A.H.; Friedrich, D.; Sarkisov, L. Automated analysis and benchmarking of GCMC simulation programs in application to gas adsorption. Mol. Simul. 2018, 44, 309-321. [CrossRef]

25. Gautam, S.; Liu, T.; Patankar, S.; Tomasko, D.; Cole, D. Location dependent orientational structure and dynamics of ethane in ZSM5. Chem. Phys. Lett. 2016, 648, 130-136. [CrossRef]

26. Thomas, A.M.; Subramanian, Y. Simulations on "Powder" Samples for Better Agreement with Macroscopic Measurements. J. Phys. Chem. C 2019, 123, 16172-16178. [CrossRef]

27. Thomas, A.M.; Subramanian, Y. Diffusion processes in a poly-crystalline zeolitic material: A molecular dynamics study. J. Chem. Phys. 2018, 149, 064702. [CrossRef] 
28. Gautam, S.; Cole, D. $\mathrm{CO}_{2}$ Adsorption in Metal-Organic Framework Mg-MOF-74: Effects of Inter-Crystalline Space. Nanomaterials 2020, 10, 2274. [CrossRef]

29. Nag, S.; Subramanian, Y. Anomalous Diffusivity in Porous Solids: Levitation Effect, Zeolites—New Challenges, Karmen Margeta and Anamarija Farkaš; IntechOpen: Rijeka, Croatia, 2020. Available online: https://www.intechopen.com/books/zeolites-newchallenges/anomalous-diffusivity-in-porous-solids-levitation-effect (accessed on 25 December 2020). [CrossRef]

30. Williams, T.; Kelley, C.; Lang, R.; Kotz, D.; Campbell, J. Gnuplot 4.6: An Interactive Plotting Program. 2014. Available online: http:/ / gnuplot.info (accessed on 25 December 2020). 